\title{
Smart Cities: alegoría del progreso en el nuevo siglo
}

\author{
Rodrigo Ramírez Autrán*
}

Recibido el 25 de febrero 2016; aceptado el 22 de septiembre de 2016

\begin{abstract}
The aim of this paper is to reflect on the concept of Smart Cities in the context of rapid urban development, and the advent of digital technologies in many social phenomena of today's global society. Some challenges and opportunities that are behind this widely used concept are analyzed. Particularly aspects from the perspective of Social Sciences arise.

Key words: Smart City, Megacities, Social Anthropology, Information and Communication Technologies, Innovation.
\end{abstract}

\section{Resumo}

O seguinte texto busca semear uma reflexão em torno do conceito de Smart Cities (Cidades Inteligentes) no contexto do desenvolvimento urbano exponencial e advento das tecnologias digitais em muitos dos fenômenos sociais da atual sociedade global. Se planejam alguns objetivos e oportunidades que estão por trás deste conceito amplamente utilizado, aspectos analisados aqui particularmente desde a ótica das Ciências Sociais.

Palavras chave: Smart City, mega cidades, Antropologia Social, Tecnologias de informação e comunicação, inovação.

\section{Resumen}

El siguiente texto busca plantear una reflexión en torno al concepto de Smart Cities (Ciudades Inteligentes) en el contexto del desarrollo urbano exponencial y el advenimiento de las tecnologías digitales en muchos de los fenómenos sociales de la

* Investigador, Centro de Investigación e Innovación en Tecnologías de Información y Comunicación -iNFOTEC, Av. San Fernando núm. 37, Col. Toriello Guerra, Ciudad de México, correos electrónicos: ramirez.autran.rodrigo@gmail.com, Rodrigo.ramirez@infotec.mx 
actual sociedad global. Se plantean algunos retos y oportunidades que están detrás de este concepto ampliamente utilizado, aspectos analizados aquí particularmente desde la óptica de las Ciencias Sociales.

Palabras clave: Smart City, mega-ciudades, Antropología Social, Tecnologías de información y comunicación, innovación.

En el siglo XXI ha florecido un proceso de urbanización a nivel mundial, el cual trajo consigo que las ciudades tengan que hacer frente a nuevos retos, como son la creciente concentración de la población, el aumento de los niveles de consumo, mayores necesidades de movilidad o el incremento de la demanda en seguridad ciudadana y de participación en las decisiones. Estos nuevos retos afectan de forma transversal a distintos ámbitos de la ciudad, por lo que su solución debe abordarse con una visión integrada e innovadora desde todas las perspectivas y áreas clave de la misma.

Para superar estos retos las ciudades se erigen como un complejo entramado de actores, donde intervienen distintas instituciones, empresas, redes, y habitantes. En dicha visión de integración urbana, para la empresa INDRA una de las multinacionales más importantes en el sector de las TIC, la necesidad más fuerte de las ciudades actualmente es evolucionar hacia un modelo de gestión de mayor calidad y eficiencia que permita:

1) Consolidar el crecimiento de la ciudad y permitir una evolución flexible y ordenada,

2) Proporcionar a los ciudadanos servicios de mejor calidad y de manera más eficiente; con un menor coste de modo que se pueda lograr una administración sostenible y

3) Obtener una visión integrada de todos los ámbitos de la ciudad de manera que se obtengan sinergias y ahorros operativos.

Como respuesta a una serie de necesidades como una mejor gestión, calidad de vida y menor costo de los servicios, surge el concepto de las Smart Cities, que encuentra su antecedente más visible en los estudios y teorizaciones que se realizaron a finales de la década de los ochenta y a lo largo de los noventa, particularmente centrados sobre el impacto de las nuevas redes de telecomunicaciones en determinados desarrollos urbanos. Algunos como Arnal, nos dicen que dicho impacto se mostró a partir "de la constatación de que las nuevas empresas de servicios avanzados parecían sensibles a la oferta de volver al centro de las ciudades - dejando atrás la época de los parques de negocios y tecnológicos de los extrarradios- si se les 
garantizaba una infraestructura comunicativa potente" (Arnal, 2012:80). En el caso europeo, por ejemplo, el desarrollo de este concepto (Arnal, 2012) estuvo estrechamente ligado al propio crecimiento del Internet a lo largo de los años noventa, particularmente en partir de la popularización de la web.

A partir de estos cambios, el éxito de nuevos modelos teóricos que empezaban a poner el énfasis no tanto en las infraestructuras como en las personas (los habitantes urbanos). Puesto que las redes empezaban a ser extendidas, el factor decisivo resultaba ser la concentración de personas capacitadas y con sentido innovador para imaginar nuevos usos de las redes. De esta forma, este fue el momento de apogeo de propuestas sobre el desarrollo de las nuevas clases creativas urbanas de autores como Richard Florida, Charles Landry y otros más "a la tecnología hay que sumar el talento, en un ambiente de tolerancia, para disparar la creatividad como fuente de creación de riqueza. Ahí estaba el secreto de las ciudades que dominarían el siglo XXI" (Achaerandio, 2001:76).

De igual forma, se ha dicho que hemos entrado a una nueva etapa de determinismo y proliferación tecnológica donde, somos testigos de una propagación (en casi todas las regiones del mundo) de proyectos urbanos designados como Smart Cities, hasta el punto que parece que de "la noche a la mañana todas las ciudades son smart". La popularidad de éste concepto experimentó un crecimiento exponencial, "eclipsando otros conceptos previos con visiones más integrales como la ciudad sostenible o aquellas otras que respondían de una manera más adecuada a la era en red y a las nuevas relaciones socioeconómicas derivadas de ella, como la ciudad del conocimiento".

Si bien ésta sobreexposición terminológica, ha atravesado profundamente en los foros profesionales e institucionales; en lo que a la ciudadanía respecta, no ha generado sino un "enorme desapego", tal y como reconocía el presidente de la Federación Española de Municipios y Provincias (FEMP) y alcalde de Santander, Iñigo de la Serna, lo cual parece establecer un indicador sobre lo alejadas que están las ciudades, autodenominadas inteligentes, de sus propios habitantes.

Hemos corroborado empíricamente (Matus y Ramírez, 2016) que el impulso de una ciudad inteligente es una tarea sumamente compleja, ya que la implementación del concepto demanda el concierto de múltiples agentes — sector público, privado, academia y ciudadanos - en ámbitos diversos para impulsar el capital físico de la ciudad, así como su capital intelectual y social (Dameri, 2014). La planificación de la ciudad no es sólo una cuestión de diseño urbano e instrumentación tecnológica, sino que además requiere de un profundo diálogo entre las ciencias sociales, la informática y las ingenierías (Ibíd.).

No hay duda que la incorporación al léxico emprendedor, científico e industrial de toda esta terminología, así como el desarrollo de agendas, propias de grandes empresas tecnológicas, han sido algunos de los detonantes primordiales de la actual 
explosión de Smart solutions que estamos viviendo. Se sabe que por lo menos CISCO, IBM, Siemens y Philips han creado estrategias para hacer cada vez más visibles sus productos y soluciones tecnológicas aplicables a los servicios urbanos, sin descartar a otras más pequeñas.

Pero, en buena medida, esas son las principales protagonistas de una estrategia de marketing novedosa (Fernández, 2012; Townsend, 2013; Borja, 2012) en muchos aspectos, sobre todo por la capacidad que han tenido de penetrar en medios generalistas con herramientas comunicativas muy significativas (secciones especiales en las ediciones en papel y digitales de los principales periódicos del mundo, páginas web propias que actúan como aglutinadoras de casos y referencias, eventos patrocinados, sistemas concursos dirigidos a ayuntamientos de todo el mundo que reciben como premio inversiones gratuitas en tecnologías, etc.). No obstante, como hemos visto no es sólo una cuestión de marketing:

Estas empresas, grandes corporaciones disponen de fuertes capacidades tecnológicas para renovar y transformar la gestión del tráfico, la tecnologización de las infraestructuras urbanas y de realizar inversiones masivas. Esto, en sí mismo, está bien. Siempre viene bien, pero plantea varias dudas. Agenda-setting o algo así le llaman en los círculos del análisis político. La capacidad de situar un tema en el centro del debate, un tema inesperado para distraer la atención o para crear un relato nuevo que sitúe nuevas prioridades y discursos alternativos. Es la sensación que tengo con la explosión del debate sobre las Smart Cities, que en los últimos tiempos aparece recurrentemente aunque lleva al menos diez años de recorrido y que, sin embargo, ahora estas empresas parecen estar capitalizando en una determinada dirección (Fernández, 2012:9).

Como lo afirmarán algunos de los consultores del CTecno (2012) aún no existe ninguna ciudad — en el mundo - con una implementación integral de Smart City. Este es el gran reto de las ciudades ya existentes como Barcelona "dado que su proceso de transformación es mucho más complejo, con impacto en la vía pública, en el mobiliario urbano y en los edificios ya construidos" (CTecno, 2012:23). Por desgracia, el foco de varios grupos dentro del movimiento Smart City es dividido:

...expertos urbanos y académicos piensan acerca de los "por qué" a una gran distancia, mientras que las empresas de tecnología y los consultores se centran en el "qué". En general, poco tiempo se ha dedicado a discutir el "cómo", que irónicamente es donde los líderes de la ciudad necesitan más ayuda. Un enfoque integral de una ciudad inteligente a última instancia, puede ayudar a resolver el "cómo" (Falconer y Mitchell, 2012:7).

Por todo ello, considero que bajo este nuevo esquema de desarrollo urbano, existe numerosas razones (necesidad de una mayor inclusión social, mayor ahorro de recursos, mayor eficiencia tecnológica, etc.) este es posiblemente, el futuro de 
muchas de las megaciudades en el mundo. Cuanto antes los gobiernos, junto con las empresas y otros agentes de todos los niveles, estén convencidos de los mayores beneficios que se pueden obtener actuando en conjunto, más rápido se promoverán y apropiarán las soluciones denominadas como inteligentes, que a pesar de todo, no son otra cosa, que respuestas a necesidades concretas, pero que de nada sirven sin la participación de los involucrados, así como la integración de los elementos sociotecnológicos, materiales, políticos y culturales necesarios.

\section{Bibliografía}

Achaerandio, Rafael et. al. (2001). "Análisis de las Ciudades Inteligentes en España, IDC Analyze the Future”, patrocinado por BBVA, Ferrovial Servicios, IBM, Microsort, Sage, Telefónica y Urbótica, en: <www.lacatedralonline.es/.../ IDCCiudadesinteligentes.pdf>, acceso: 17 de diciembre de 2012.

Arnal, José Carlos (2012). "Smart cities: oportunidad económica y desafío urbano", Revista Economía Aragonesa, núm. 48, septiembre, iberCaja Banco S.A.U.

Borja, Jordi (2003). La ciudad conquistada, Editorial Alianza, Madrid.

Ctecno (2012). Hoja de Ruta para la Smart City Barcelona, febrero, CercleTecnologic de Cataluyna.

Dameri, Renata (2014). Smart City How to Create Public and Economic Value with High Technology in Urban Space, Springer, New York.

Falconer, Gordon y Shane Mitchell (2012). Smart City Framework. A Systematic Process for Enabling Smart+Connected Communities, September, Cisco Internet Business Solutions Group (IBSG).

Fernández, Manu (2012). "Smart City. Tecnologías emergentes para el funcionamiento urbano", julio 2011, Naider, en:

$<$ http://www.scribd.com/doc/61950985/Smart-City-Tecnologias-emergentespara-el-funcionamiento-urbano $>$.

Matus y Ramírez (coords.) (2016). Ciudades inteligentes en Iberoamérica; ejemplos de iniciativas desde el sector privado, la sociedad civil, el gobierno y la academia, Infotec, México.

Townsend, Anthony (2013). Smart Cities: Big Data, Civic Hackers, and the Quest for a New Utopia, WW Norton \& Company, New York. 